\title{
On a Sensitivity-Analysis-Based Design Method for Shearer Parts
}

\author{
Qiu-Xia Fan and Zhao-Jian Yang* \\ College of Mechanical Engineering, Taiyuan University of Technology, Taiyuan, 030024, China
}

\begin{abstract}
To improve the repeatability of CAE analysis and design on shearer parts, the author proposes a static-dynamic integration design method for shearer parts while taking into account their structural features. The idea behind this method is that the design is analysis-driven and together with network technology, parameterized technology and optimization technology, it leads to conceptual design of shearer parts and through the integrated analysis on sensitivity of shearer parts in both static and dynamic states, the best design scheme can be offered to users. The author verifies the feasibility and effectiveness of this method by analyzing here the conceptual design of the planet carrier in the cutting unit of some shearer.
\end{abstract}

Keywords: Conceptual design, CAD, Sensitivity, Shearer, Static-dynamic, Dynamic property.

\section{INTRODUCTION}

The development of shearers, which are known for their wide range of varieties and parts [1,15], is typical knowledge-intensive work [2]. CAE design features lack of repeatability and experts worldwide have dedicated themselves to the research of repeatable models to improve repeatability and analysis efficiency of CAE design. Zhang Shanhui [3] from Shandong University and his team proposed in 2010 an ontology-based knowledge-embedding design method for mechanical products to address the limited repeatability of their design materials. Luo Shijian and Zhu Shangshang [4] came up with an ontology-based product design DNA method to tackle search inaccuracy in industrial design schemes. Jiang Yang [5] and his colleagues from Ha'erbin Industrial University created a knowledge integrator based on the ontology of design knowledge to solve the problem of knowledge redundancy and result optimization in knowledge integration of complicated products. Cao Dongxing [6], a professor with Hebei Industrial University constructed the conceptual design process of port ontology. The researches above are all conducted from the angle of model repeatability and fail to solve the problem for good.

In the past few years, some experts have tried to handle this deficiency from its root conceptual design. Chen Xuling and Lou Peihuang [7] put forward a mapping method that mixed behavior-function and behavior structure in the conceptual design of industrial products; Liu Haiqiang [8] and his team from Zhejiang University created a modelbuilding method that combines Petri net extension and NSGA algorithm in conceptual design of complicated products. Zhang Wei and his co-workers applied neural net to the conceptual design of helicopters [9]. The researches above are carried out from a single aspect of conceptual

*Address correspondence to this author at the College of Mechanical Engineering, Taiyuan University of Technology, Yingze Street, Taiyuan, 030024, China; Tel: 13834511099; E-mail: yangzhaojian@tyut.edu.cn design and fail to cover the entire conceptual design. Ding Hua from Taiyuan University of Technology proposed in 2010, an integrated model based on theories of rough set and support vector machines and applied it to the conceptual design of the entire machine of shearers [10]. However, the conceptual design of shearers is a systematic project which involves detailed conceptual design on the whole machine and its parts so as to avoid repeated design in the following research. On such basis, the author here proposes a conceptual design method based on sensitivity algorithm under both static and dynamic conditions [16], and finds out the optimal solution in the phase of conceptual design combining structural design, analytic computation and optimization design so as to reduce the repeatability coefficient in the following research and shorten the design duration and lower the cost.

\section{STATIC-DYNAMIC INTEGRATION ALGORITHM FOR SHEARER PARTS}

The key to optimization of static-dynamic integration is to allocate the weight among various static and dynamic levels. The author combines planning and average frequency method and proposes an integrated optimized algorithm that takes into account the structural rigidity and multi-level modal of shearer parts.

Static multi-level optimized objective function is as follows:

$$
\begin{aligned}
& \min C(\rho)=\left(\omega_{1} \frac{C_{1}(\rho)-C_{1, \text { min }}}{C_{1, \text { max }}-C_{1, \text { min }}}+\omega_{2} \frac{C_{2}(\rho)-C_{2, \text { min }}}{C_{2, \text { max }}-C_{2, \text { min }}}\right. \\
& \left.+\cdots+\omega_{m} \frac{C_{m}(\rho)-C_{m, \text { min }}}{C_{m, \text { max }}-C_{m, \text { min }}}\right)
\end{aligned}
$$

In which, $C_{1}(\rho), C_{2}(\rho), C_{m}(\rho)$ are flexibility values in various working conditions, $\omega$ is the weight of 
flexibility objective function and $C_{m, \max }$ and $C_{m, \min }$ are the maximum and minimal of flexibility objective function in working condition $m$.

Average frequency method is used to study multi-level optimization of dynamic natural frequency and objective function of modal multi-level optimization as follows:

Comprehensive objective function of multi-level optimization is as follows:

$\max \wedge(\rho)=\lambda_{0}+s\left(\frac{\varphi_{1}}{\lambda_{1}-\lambda_{0}}+\frac{\varphi_{2}}{\lambda_{2}-\lambda_{0}}+\cdots+\frac{\varphi_{n}}{\lambda_{n}-\lambda_{0}}\right)$

In which, $\wedge(\rho)$ is average frequency, $\lambda_{1}, \lambda_{2}$ and $\lambda_{n}$ are modal vibration frequencies at each level, $\lambda_{0}$ and $s$ are the coefficients to adjust objective function, and $\varphi_{1}, \varphi_{2}$ and $\varphi_{n}$ are weight coefficients of modal vibration frequency at each level.

As for the optimized objective which takes into account the rigidity and modal of shearer parts, its optimization objective function of static-dynamic integration is as follows:

$$
\begin{aligned}
& \min F(\rho)=\left[\omega^{2}\left(\sum_{k=1}^{m} \frac{C_{k}(\rho)-C_{k, \text { min }}}{C_{k, \text { max }}-C_{k, \text { min }}}\right)^{2}+\right. \\
& \left.\left(1-\omega^{2}\right)\left(\frac{\wedge_{\text {max }}-\wedge(\rho)}{\wedge_{\text {max }}-\wedge_{\min }}\right)^{2}\right]^{\frac{1}{2}}
\end{aligned}
$$

In which $F(\rho)$ is comprehensive objective function for multi-level optimization, and $\wedge_{\max }$ and $\wedge_{\min }$ are the maximum and minimum of frequency objective function.

\section{ANALYSIS ON STATIC-DYNAMIC SENSITIVITY OF SHEARER PARTS}

Sensitivity analysis on shearer parts is one that analyzes the various optimized results of the chosen ideal scheme, which includes static sensitivity analysis and dynamic sensitivity analysis.

\subsection{Sensitivity Analysis on Dynamic Property}

Sensitivity of dynamic properties of shearer parts refers to the sensitivity of frequency, i.e. the sensitivity of natural frequency to shearer design variables [11-13].

In modal analysis, the natural frequency property of system vibration is defined as $\left(K-\omega^{2} M\right) \delta=0$, in which M is the mass of the system, $\mathrm{K}$ the rigidity matrix and $\delta$ is the natural frequency.

Taking the partial derivative of design variable $x_{i}$, we get

$$
\frac{\partial K}{\partial x_{i}} \delta+K \frac{\partial \delta}{\partial x_{i}}-\frac{\partial \omega^{2}}{\partial x_{i}} M \delta-\omega^{2} \frac{\partial M}{\partial x_{i}} \delta-
$$

$\omega^{2} M \frac{\partial \delta}{\partial x_{i}}=0$

Multiplying equation (1) with $\delta^{T}$ on the left and then we can get

$\frac{\partial \omega^{2}}{\partial x_{i}} \delta^{T} M \delta=\delta^{T} \frac{\partial K}{\partial x_{i}} \delta-\omega^{2} \delta^{T} \frac{\partial M}{\partial x_{i}} \delta+\left(K \delta-\omega^{2} M \delta\right)^{T} \frac{\partial \delta}{\partial x_{i}}$

From equation (1) we can have $\left(K \delta-\omega^{2} M \delta\right)^{T}=0$.

Simplifying equation (2), we have

$\frac{\partial \omega^{2}}{\partial x_{i}}=\frac{\delta^{T} \frac{\partial K}{\partial x_{i}} \delta-\omega^{2} \delta^{T} \frac{\partial M}{\partial x_{i}} \delta}{\delta^{T} M \delta}$

Because of $\omega=2 \pi f$, the sensitivity of vibration natural frequency to design variable can be obtained:

$\frac{\partial f}{\partial x_{i}}=\frac{1}{8 \pi^{2} f} \delta^{T} \frac{\partial K}{\partial x_{i}} \delta-\frac{f}{2} \delta^{T} \frac{\partial M}{\partial x_{i}} \delta$

From equation (3) the formula to calculate the sensitivity of the natural frequency to structural parameter can be obtained as:

$$
\frac{\partial f}{\partial h}=\sum_{m} \delta_{e}^{T}\left[\frac{1}{8 \pi^{2} f}\left(E K_{p e}^{e}+3 E h^{2} K_{b e}^{e}\right)-\frac{f}{6} \rho A_{e} M_{c}^{e}\right] \delta_{e}
$$

In which, $f$ is the natural frequency of the system, $\mathrm{E}$ the modulus of material elasticity, $h$ unit thickness, $K_{p e}^{e}$ and $K_{b e}^{e}$ the part irrelevant to $\mathrm{E}$ and $h$ in rigidity matrix, $M_{c}^{e}$ the part irrelevant to $h$ in mass matrix, $\rho$ material density, $A_{e}$ unit superficial area, $m$ the number of shell unit with thickness $m$ and $\delta_{e}$ the feature vector for the component of unit $e$ with thickness $h$.

\subsection{Sensitivity Analysis on Static Property}

Static sensitivity of shearers refers to sensitivity to node displacement. Balance equation of shearer structure is $K \delta=F[14]$, in which $\mathrm{K}$ is shearer rigidity matrix, $\mathrm{F}$ shearer load vector and $\delta$ node displacement vector.

Sensitivity of node displacement can be calculated through

$$
\frac{\partial \delta}{\partial h}=\frac{\Delta \delta}{\Delta h}=\frac{\delta(h+\Delta h)-\delta(h)}{\Delta h}
$$

\subsection{Static-Dynamic Integration Design of Shearers}

The design is conducted on Windows XP with Internet Information Server as Web server. Developers seamlessly integrate ANSYS by combining network development technologies of JSP and Java Beans to perform analysis optimization iteration. Through static-dynamic sensitivity analysis, the optimal scheme is found and the integrated 
design of structural rigidity and multi-level modals of the conceptual model of shearer parts are estimated. The flow chart of static-dynamic integration design is shown in Fig. (1).

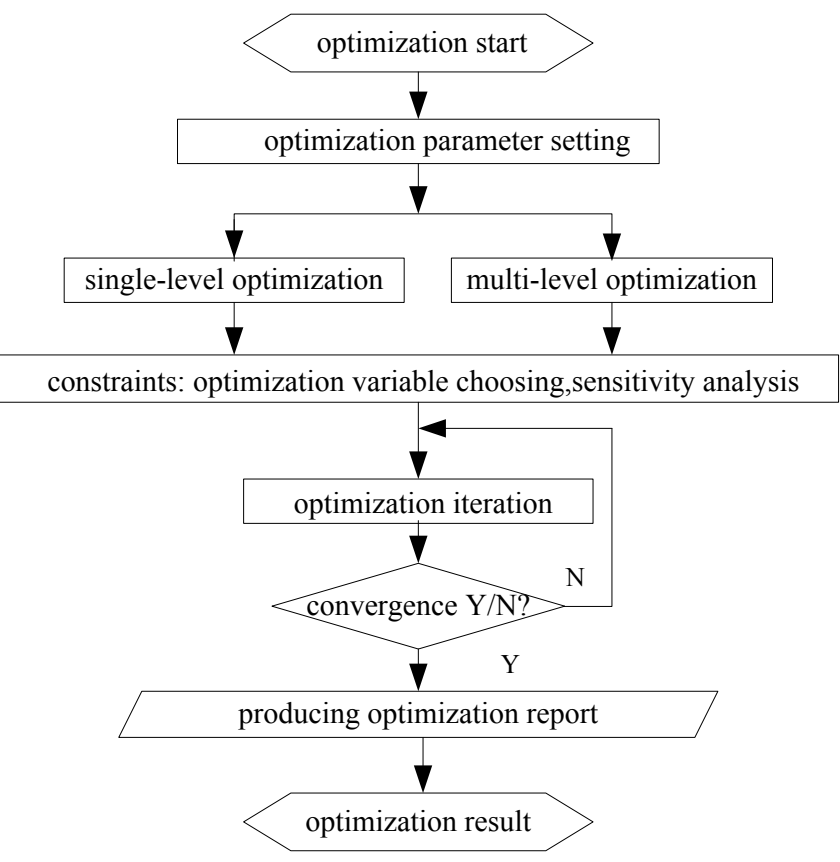

Fig. (1). Flow chart of static-dynamic integration design.

\section{CASE STUDY}

To verify the feasibility of the method mentioned above, the author applies the sensitivity-based static-dynamic integration design to the conceptual design of the planet carrier of shearer cutting unit and finds out the optimal scheme (Fig. 2).

Parameters used in this test are as follows:

Output power of cutting unit motor: $750 \mathrm{~kW}$

Output rotational speed of cutting unit motor: $1480 \mathrm{r} / \mathrm{min}$

Torque load M=72330N-m

Step 1: through case search based on design and evaluation indicators (methods of case search are not introduced here due to the length of the paper), four conceptual design schemes are proposed as shown in Table 1, with parametric variables: $\mathrm{LA}, \mathrm{LB}, \mathrm{LC}, \mathrm{LD}, \mathrm{DA}, \mathrm{DB}, \mathrm{DC}, \mathrm{DH}$ as shown in Fig. (3).

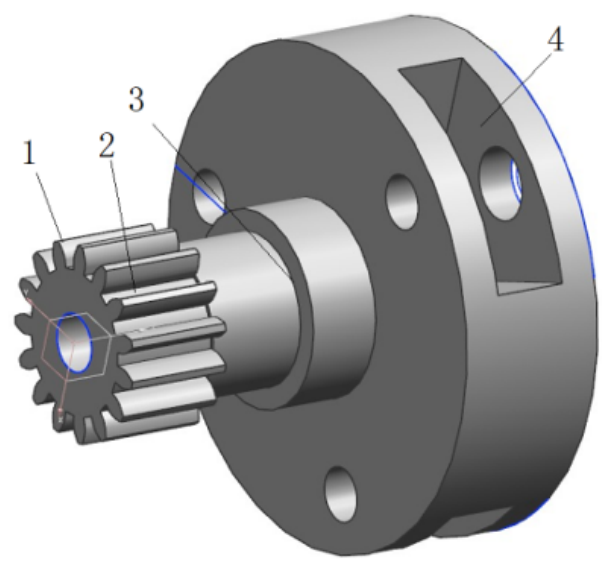

Fig. (2). The planet model of shearer cutting unit.

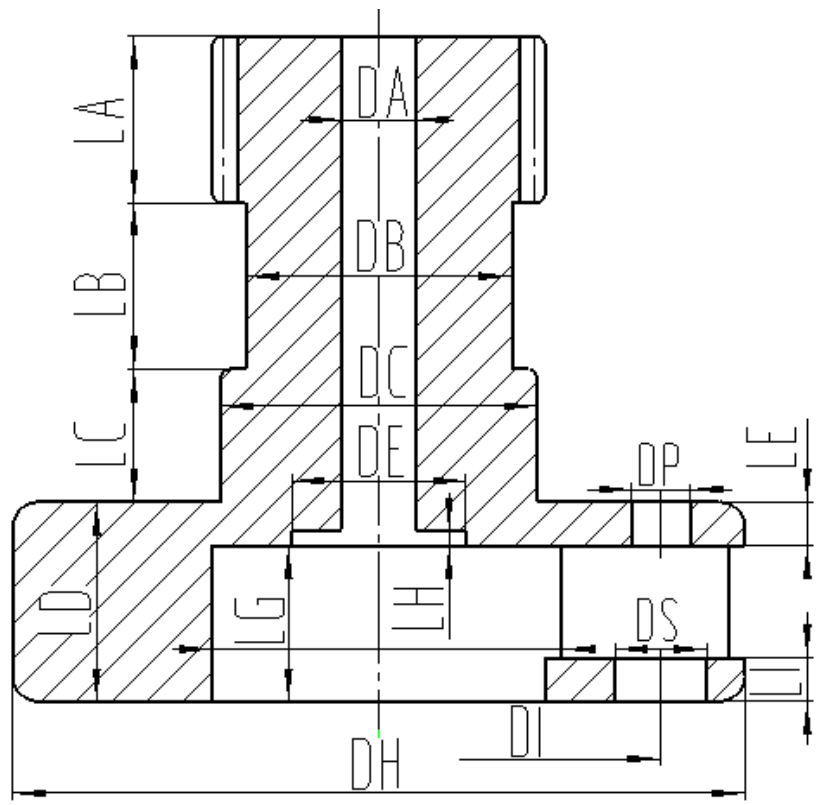

Fig. (3). Variable position.

The mass of planet carrier has been reduced respectively by $28.8 \mathrm{~kg}, 7 \mathrm{~kg}, 24.4 \mathrm{~kg}$ and $34.9 \mathrm{~kg}$ from Scheme 1 to Scheme 4. Thus, Scheme 4 is the optimal one.

Table 1. Optimization scheme.

\begin{tabular}{|c|c|c|c|c|c|}
\hline mass $/ \mathrm{kg}$ & 689.2 & 660.4 & 682.2 & 664.8 & 654.3 \\
\hline $\mathrm{LB} / \mathrm{mm}$ & 175 & 140 & 170 & 135 & 130 \\
\hline $\mathrm{LC} / \mathrm{mm}$ & 125 & 100 & 120 & 115 & 100 \\
\hline $\mathrm{DA} / \mathrm{mm}$ & 85 & 80 & 80 & 75 & 75 \\
\hline $\mathrm{DB} / \mathrm{mm}$ & 245 & 210 & 240 & 205 & 205 \\
\hline $\mathrm{DC} / \mathrm{mm}$ & 295 & 270 & 290 & 265 & 270 \\
\hline $\mathrm{DH} / \mathrm{mm}$ & 715 & 690 & 710 & 685 & 680 \\
\hline
\end{tabular}


Table 2. Contribution rate the mass makes to other performance parameters.

\begin{tabular}{|c|c|c|c|c|}
\hline & Scheme 1 & Scheme 2 & Scheme 3 & Scheme 4 \\
\hline \hline Bending rigidity & 1.1 & 0.96 & 1.05 & 0.07 \\
\hline Modal 1 & 0.38 & 0.27 & 0.1 & 0.24 \\
\hline Modal 2 & 0.46 & 0.13 & 0.40 & 0.27 \\
\hline Modal 3 & 0.37 & 0.23 & 0.41 & 0.34 \\
\hline Modal 4 & 0.57 & 0.47 & 0.1 & 0.31 \\
\hline Modal 5 & 0.27 & 0.14 & 0.25 & 0.17 \\
\hline Modal 6 & 0.31 & 0.27 & & \\
\hline
\end{tabular}

Step 2: calculating the contribution rate the shearer planet carrier makes to performance parameters, the results are shown in Table 2. Contribution rate in Scheme 4 is lower while that in Scheme $\mathbf{1}$ is higher and the mass reduction is only inferior to that in Scheme 4, so Scheme 1 is the ideal revised scheme.

Step 3: performing sensitivity analysis on the chosen ideal scheme. The results of sensitivity analysis on design variables are shown in Fig. (4) and Scheme $\mathbf{1}$ is the optimal one.

Table 3 is the design result of planet carrier optimization scheme 1 , in which the mass of planet carrier is reduced by about $28.8 \mathrm{~kg}$ from the original $689.2 \mathrm{~kg}$ to $660.4 \mathrm{~kg}$ and the planet carrier rigidity is $0.175 \mathrm{~mm}$ lower than the $0.2 \mathrm{~mm}$ optimization constraint. This optimization result has been confirmed by the client and put into practice.

This scheme has been applied among shearer manufacturers and user reports show that through the conceptual design gained from this scheme, the follow-up designs are reduced and the error rate is lowered. Design results are reliable to make up the deficiency in the traditional design.

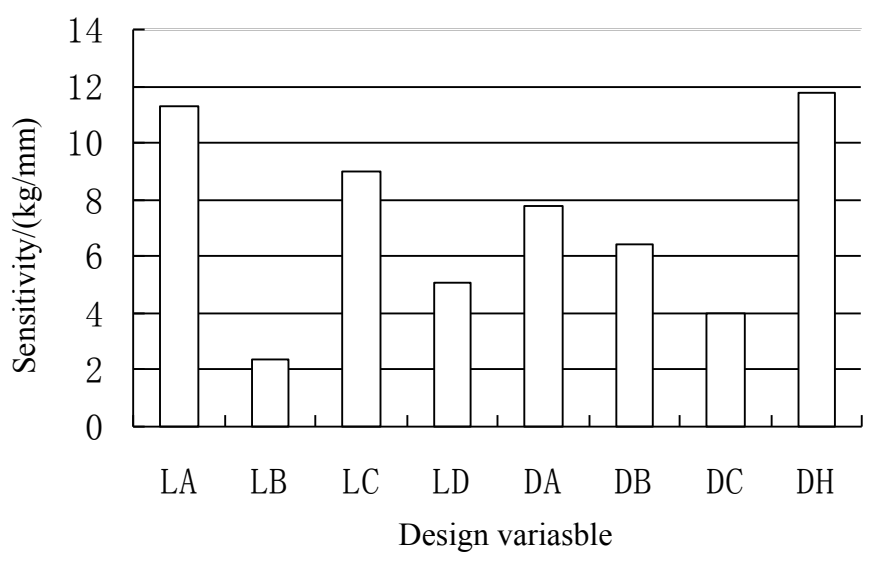

Fig. (4). Sensitivity analysis.

\section{CONCLUSION}

(1) With analysis-driven design as its ideal, this method can be easily and conveniently applied to the conceptual design of shearer parts and produce the optimal design parameters and analysis result to provide the client with the optimal design scheme and reduce the number of repetitions of the follow-up design;
(2) The author proposes dynamic sensitivity algorithm and produces sensitivity formula of the natural frequency to design variables and demonstrates its validity through case study;

(3) The author proposes static-dynamic integration optimization algorithm and applies it to this method. Through comparison of test results, the author verifies the results to be valid and the client confirms after use that it is easy to operate and that it is convenient for designers who are not familiar with professional analysis software;

(4) This paper is primarily based on the conceptual design of shearer parts and the method in this paper can be extended to the conceptual design of other mining machinery.

Table 3. The results of optimization design.

\begin{tabular}{|c|c|c|}
\hline Optimization Items & Original Value & Optimized Value \\
\hline \hline Mass of planet carrier/kg & 689.2 & 660.4 \\
\hline $\mathrm{LA} / \mathrm{mm}$ & 185 & 150 \\
\hline $\mathrm{LB} / \mathrm{mm}$ & 175 & 140 \\
\hline $\mathrm{LC} / \mathrm{mm}$ & 125 & 100 \\
\hline $\mathrm{LD} / \mathrm{mm}$ & 245 & 210 \\
\hline $\mathrm{DA} / \mathrm{mm}$ & 85 & 80 \\
\hline $\mathrm{DB} / \mathrm{mm}$ & 245 & 210 \\
\hline $\mathrm{DC} / \mathrm{mm}$ & 295 & 270 \\
\hline $\mathrm{DH} / \mathrm{mm}$ & 715 & 690 \\
\hline
\end{tabular}

\section{CONFLICT OF INTEREST}

The authors confirm that this article content has no conflict of interest.

\section{ACKNOWLEDGEMENTS}

This paper belongs to the project of the "Key Technology Special Subjects Foundation of Shanxi Province", No. 20111101040; and "Youth Science and Technology Research Fund of Shanxi Province", No. 2012021022-6; and "Youth Team Startup Project of Taiyuan University of Technology”, No. 1205-04020102. 


\section{REFERENCES}

[1] S. Zhiyuan, Z. Zhencai, and H. Zhenduo, "The discussion on mining-Machinery development". Mining \& Metallurgy, vol. 15, pp. 86-9, 2006.

[2] H. HUBEL, and G.J. COLQUH, "A reference architecture for engineering data control (EDC) in capital plant manufacture", Computers in Industry, vol. 46, pp. 149-65, 2001.

[3] Z. Shanhui,Y. Chaoying, and L. Zhenyu, "Knowledge embedding method for mechanical product design based on ontology", Computers in Industry, vol. 16, pp. 2385-91, 2010.

[4] L. Shijian, and Z. Shangshang, "Ontology based product family design DNA in industrial design", Computers in Industry, vol. 15, pp. 226-33, 2009.

[5] J. Yang, J. Tianguo, L. Wenjian, and X. Jie, "Ontology based optimization integration for design knowledge of complicated product", Computers in Industry, vol. 16,pp. 1828-35, 2010.

[6] C. Dongxing, "Port-based ontology conceptual design theory", Journal of Mechanical Engineering, vol. 46, pp. 123-32, 2010.

[7] C. Xuling, L. peihuang et al., "Behavior- function \& Behaviorstructure hybrid mapping in mechanical concept design", Computer Integrated Manufacture Systems, vol. 16, pp. 1363-70, 2010.

[8] L. Haiqiang, Q. guoning, Z. Taihua, and J. Yangjian, "Research on multi-disciplinary process modeling of concept design for complex product", Journal of Zhejiang University, vol. 43, pp. 517-22, 2009.
[9] S. Weiand, and Z. Chenglin, "Application of neural networks in helicopter conceptual design", Journal of Nanjing University of Aeronautics \& Astronautics, vol. 41, pp. 121-5, 2009.

[10] D. Hua, and Y. Zhaojian, "Research and practice of shearer conceptual design fusion reasoning model", Journal of China coal society, vol. 35,2010 , pp. $1748-53$.

[11] F. Giunchiglia, Contextual reasoning, Proceedings IJCAIp93 Workshop on using knowledge in its context. Chambery, France, 1993, pp. 39-49.

[12] J. Yuncheng, S. Zhongzhi, T. Yong, and W. Ju, "A distributed dynamic description logic", Journal of Computer Research and Development, vol. 43, pp. 1603-8, 2006.

[13] I. Horrocks, "The FaCT system", Proceedings of TABLEAUX-98 Oisterwijk, Netherlands, 1998, pp. 307-12.

[14] P.D.T. O'Connor, "Practical Reliability Engineering", $4^{\text {th }}$ Beijing: Publishing house of electronics industry, 2005, pp. 20-21.

[15] H. Gao, and Z. Yang, "Computer dynamic simulation of double cylinder shearer's cutting unit on the opposing rolling mode", AISS, vol. 5, pp. 734-45, 2013.

[16] H. Yin, B.-Z. Li, T.Guo, and J.-X. Zhu, "Analysis model and empirical research on product innovation process of manufacturing enterprises based on entropy-topsis method", Advances in Information Sciences and Service Sciences, vol. 5, pp. 637-44, 2013.

This is an open access article licensed under the terms of the Creative Commons Attribution Non-Commercial License (http://creativecommons.org/licenses/ by-nc/4.0/) which permits unrestricted, non-commercial use, distribution and reproduction in any medium, provided the work is properly cited. 